(C) Journal of Applied Mathematics \& Decision Sciences, 1(2), 119-130 (1997)

Reprints Available directly from the Editor. Printed in New Zealand.

\title{
ON THE SIGNIFICANCE LEVEL OF THE MULTIRELATION COEFFICIENT †
}

\author{
ROGER DEAR AND ZVI DREZNER \\ Department of Management Science/Information Systems \\ California State University-Fullerton Fullerton, CA 92834 USA.
}

\begin{abstract}
The concept of the multirelation coefficient is defined to describe the closeness of a set of variables to a linear relation. This concept extends the linear correlation between two variables to two or more variables. Parameters of a beta distribution are determined that are utilized to approximate significance levels of the multirelation coefficient for any given number of observations and variables. A generalized Student $t$ distribution is defined. This distribution, which is termed the multirelated $t$ distribution, reduces to the Student $t$ distribution for two variables. It is useful in the determination of the significance level of the multirelation coefficient.
\end{abstract}

Keywords: Multirelation, Multiple correlation, Multiple regression.

\section{Introduction}

The concept of the multirelation coefficient is described in Drezner (1995). It gives a measure of closeness of a set of variables to a linear relationship. In order to be able to compare the significance level of two multirelation coefficients with different dimensionality, the significance level of the multirelation coefficient is needed. We evaluate the distribution of the multirelation coefficient and its fractiles for a finite number of observations. There might be ways to evaluate the limit of the distribution as the number of observations increases to infinity. Such a result might have some practical interest but is not investigated here.

First, the concept of multirelation coefficient is introduced and then some of its properties are outlined (for a detailed discussion see Drezner (1995)). Let $\lambda(R)$ be the least eigenvalue of the correlation matrix $R$ between a given set of $k$ variables. The multirelation coefficient $r\left(Y_{1}, \ldots, Y_{k}\right)$ is defined as: $r\left(Y_{1}, \ldots, Y_{k}\right)=1-\lambda(R)$. The multirelation coefficient is a measure of the linear relation among all the $Y_{i}$ for $i=1, \ldots, k$.

The following properties are proven in Drezner (1995) and help explain the role and the properties of the multirelation coefficient.

Property $10 \leq r\left(Y_{1}, \ldots, Y_{k}\right) \leq 1$.

Property $2 r\left(Y_{1}, \ldots, Y_{k-1}\right) \leq r\left(Y_{1}, \ldots, Y_{k}\right)$.

$†$ Part of this research was done while the second author was on sabbatical leave at the Hong Kong University of Science and Technology, Kowloon, Hong Kong. 
Property $3 r\left(Y_{1}, \ldots, Y_{k}\right)=0$ iff $r_{i j}=0$ for every $1 \leq i<j \leq k$.

Property $4 r\left(Y_{1}, \ldots, Y_{k}\right)=1$ iff some vector is a linear combination of the other vectors. (Or, in other words, the vectors are linearly dependent).

\section{On the Distribution of the Multirelation Coefficient}

In order to be able to compare multirelation coefficients with different number of variables and observations the fractiles of the multirelation coefficient are helpful. In this section we approximately calculate these fractiles for a given number of observations and variables.

First, some properties of the eigenvalues of the correlation matrix are found. Let, for a given correlation matrix $R=\left\{r_{i j}\right\}$,

$$
\bar{r}=\sqrt{\sum_{i \neq j} \frac{r_{i j}^{2}}{k(k-1)}}
$$

The eigenvalues of $R$ are $\lambda_{1}, \ldots, \lambda_{k}$. By matrix theory:

$$
\begin{aligned}
\sum_{i=1}^{k} \lambda_{i} & =\sum_{i=1}^{k} r_{i i}=k \\
\sum_{i \neq j} \lambda_{i} \lambda_{j} & =\sum_{i \neq j}\left\{r_{i i} r_{j j}-r_{i j}^{2}\right\}=k(k-1)\left(1-\bar{r}^{2}\right) \\
\operatorname{By}(2): \sum_{i=1}^{k} \lambda_{i}^{2} & =\left[\sum_{i=1}^{k} \lambda_{i}\right]^{2}-\sum_{i \neq j} \lambda_{i} \lambda_{j}=k^{2}-k(k-1)\left(1-\bar{r}^{2}\right)=k+k(k-1) \bar{r}^{2} .
\end{aligned}
$$

Therefore, we have the identities:

$$
\begin{aligned}
\mu \equiv \frac{1}{k} \sum_{i=1}^{k} \lambda_{i} & =1 \\
\sigma^{2} \equiv \frac{1}{k} \sum_{i=1}^{k} \lambda_{i}^{2}-\left[\frac{1}{k} \sum_{i=1}^{k} \lambda_{i}\right]^{2} & =(k-1) \bar{r}^{2} \\
\frac{1}{k(k-1)} \sum_{i \neq j} \lambda_{i} \lambda_{j}-\left[\frac{1}{k} \sum_{i=1}^{k} \lambda_{i}\right]^{2} & =-\bar{r}^{2} \\
\rho \equiv \frac{1}{k(k-1)} \sum_{i \neq j} \lambda_{i} \lambda_{j}-\left[\frac{1}{k} \sum_{i=1}^{k} \lambda_{i}\right]^{2} & =-\frac{1}{k-1}
\end{aligned}
$$


Assume that the elements of $k$ vectors $Y_{i}$ for $i=1, \ldots, k$ of length $n$ each have a given random distribution. By (6) the eigenvalues are a sample from a multivariate distribution $\Psi$. The correlation matrix of the sample has off diagonal elements equal to $-\frac{1}{k-1}$. This correlation matrix is singular. By (3) the means of the multivariate distribution $\Psi$ are all equal 1. It is known, (Kendall, 1980), that $E\left(r_{i j}^{2}\right)=\frac{1}{n-1}$ for any distribution of independent $Y_{i}^{\prime}$ s. Therefore, $E\left(\bar{r}^{2}\right)=\frac{1}{n-1}$. By (4) the variances of $\Psi$ are all equal to $\frac{k-1}{n-1}$.

In trying to determine the type of the multivariate distribution $\Psi$, when the $Y_{i}^{\prime}$ s are drawn from i.i.d. normal distributions, we first checked whether $\Psi$ can be approximated by a multivariate normal distribution (see the Appendix for computational details). We observed that the multivariate normal distribution is not a good approximation for the distribution of the eigenvalues and thus cannot be used to accurately derive the distribution of the multirelation coefficient. In order to find a better approximation for $\Psi$ we plotted the simulation results. In Figure 1 we present the distribution of the least eigenvalue that was obtained by calculating the eigenvalues of a correlation matrix of 5 by 5 generated by randomly generated vectors of 100 elements each. The figure shows the frequency of eigenvalues in segments of size 0.01 (i.e., between 0 and $0.01,0.01$ and 0.02 and so on) based on 100,000 correlation matrices. This distribution is not a normal distribution, nor is it symmetric. A discussion of the distribution of the eigenvalues of the correlation matrix can be found in Kendall and Stuart $(1966)^{1}$. However it does not address our particular issue of the distribution of the smallest eigenvalue. Moreover, since the mean of all eigenvalues is 1 (3), the least eigenvalue cannot exceed 1 . The distribution of the least eigenvalue is between 0 and 1 . We therefore attempted to estimate the probability density function of the least eigenvalue in order to be able to calculate the significance level of the multirelation coefficient.

The case $k=2$ can be explicitly solved. For $k=2$ the multirelation coefficient is the absolute value of the correlation coefficient. The correlation coefficient is related to the Student $t$ distribution by the relationship:

$$
t_{n-2}=\frac{r \sqrt{n-2}}{\sqrt{1-r^{2}}}
$$

The Student $t$ distribution is related to the beta distribution by the following formula (Abramowitz and Stegun, 1972):

$$
t_{\nu}=I_{x}\left(\frac{\nu}{2}, \frac{1}{2}\right) \quad \text { for } \quad x=\frac{\nu}{\nu+t^{2}}
$$

Since $\nu=n-2$, equation (7) yields:

$$
\frac{\nu}{\nu+t^{2}}=1-r^{2}
$$

Comparing (9) to (8) we get that $1-r^{2}$ is distributed by a beta distribution with parameters $a=\frac{n-2}{2}$ and $b=\frac{1}{2}$. Since $I_{1-x}(a, b)=I_{x}(b, a)$, for $k=2: r^{2}$ is 


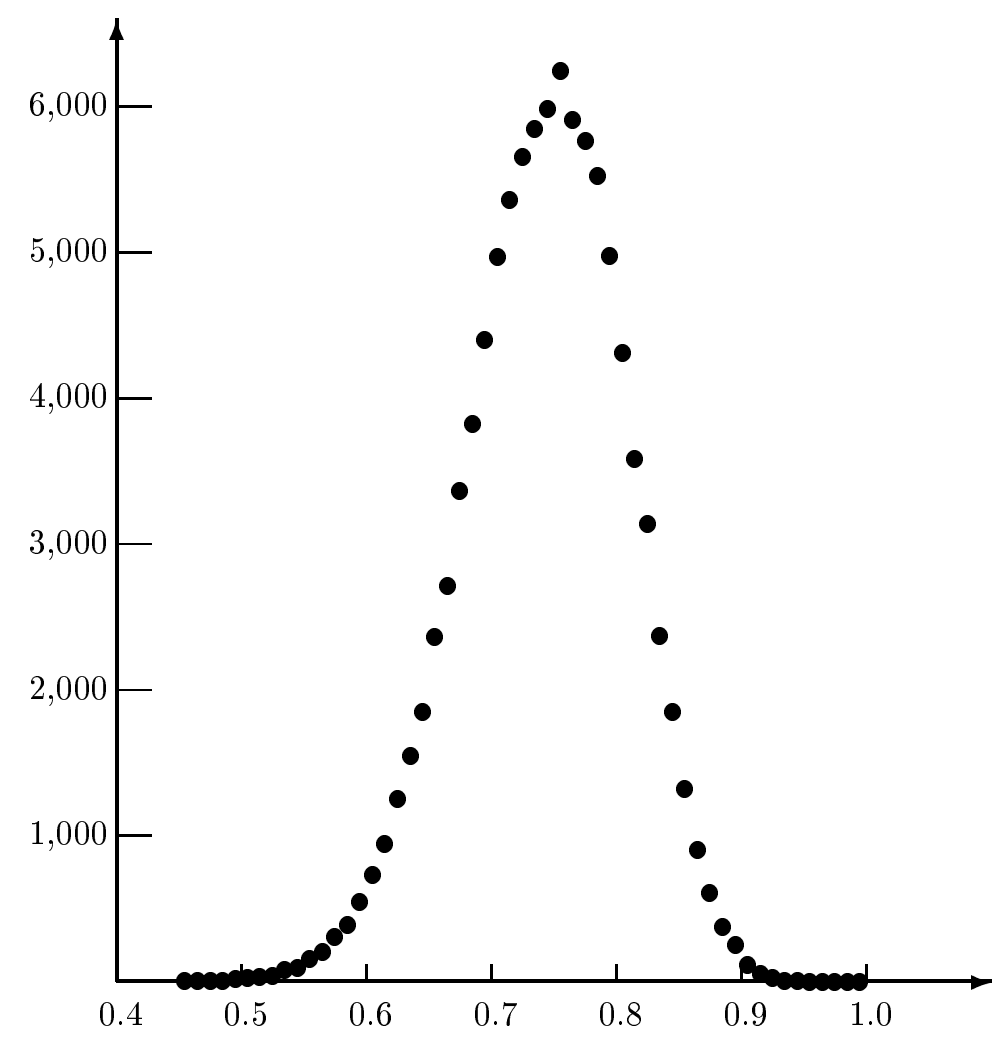

Figure 1. The distribution of the smallest eigenvalue

distributed according to a beta distribution with parameters $a=\frac{1}{2}$ and $b=\frac{n-2}{2}$. In conclusion, for the case $k=2$ the square of the multirelation coefficient is distributed according to a beta distribution.

Examination of many graphs of the distribution of the least eigenvalue led us to conclude that a beta distribution may be used to estimate the multirelation coefficient distribution or its square. In Figure 2 the frequency of the least eigenvalue is compared with the beta distribution with the mean and the variance of the simulated values. The fit justifies the exploration of the beta distribution as an approximation to the distribution of the least eigenvalue. However, since $r^{2}$ is actually a beta distribution for $k=2$, and the square of the multiple correlation coefficient is also a beta distribution with $a=\frac{k-1}{2}$ and $b=\frac{n-k}{2}$ (Stuart and Ord, 1991; Kendall and Stuart, 1966), we investigated a possible fit of a beta distribution to the distribution of $r^{2}$ rather than $r$. 




Figure 2. Distribution of the smallest eigenvalue and its beta approximation

\section{Determining the Parameters of the Beta Distribution}

In order to be able to apply the beta distribution for the calculation of the probabilities involving the multirelation coefficient, estimates for the parameters $a$ and $b$ of the distribution are required for given $k$ and $n$ (rather than be estimated by simulation). We calculated these parameters for $k=2,3, \ldots, 10$ and $n=10,20, \ldots, 100$ using only pairs $k, n$ for which $n>3 k$. The simulation was performed as follows. For a given $k$ and $n$, a matrix of size $k$ by $n$ is generated using standard generation techniques (Law and Kelton (1991), Marse and Roberts (1983)). The elements of this matrix are drawn from a standard normal distribution. The correlation matrix is calculated and the multirelation coefficient found.

For each case we simulated 20 sets of 50,000 matrices each for a total of one million matrices for each result in Table 1 . In the table we report the mean and standard error (standard deviation of the 20 sets divided by $\sqrt{20}$ ) for $a$ and $b$ calculated for each pair of $k$ and $n$. A curve fitting using multiple regression was performed on these means. Since regression analysis assumes uniform variance for all points, we regressed on $\frac{a-0.5}{k-2}$ for $a$, and $\frac{b}{n-2}-0.5$ for $b$ using only the points for $k \geq 3$. These 
Table 1. Means and standard errors of the beta parameters

\begin{tabular}{|c|c|c|c|c|c|c|c|c|c|c|c|}
\hline \multirow[b]{2}{*}{$k$} & \multirow[b]{2}{*}{$n$} & \multicolumn{2}{|c|}{$a$} & \multicolumn{2}{|c|}{$b$} & \multirow[b]{2}{*}{$k$} & \multirow[b]{2}{*}{$n$} & \multicolumn{2}{|c|}{$a$} & \multicolumn{2}{|c|}{$b$} \\
\hline & & Mean & $\begin{array}{l}\text { Std. } \\
\text { Err. }\end{array}$ & Mean & $\begin{array}{l}\text { Std. } \\
\text { Err. }\end{array}$ & & & Mean & $\begin{array}{l}\text { Std. } \\
\text { Err. }\end{array}$ & Mean & $\begin{array}{l}\text { Std. } \\
\text { Err. }\end{array}$ \\
\hline 2 & 10 & .5002 & .0009 & 4.0102 & .0089 & 6 & 30 & 6.0431 & .0074 & 13.6086 & .0178 \\
\hline 2 & 20 & .4984 & .0007 & 8.9728 & .0209 & 6 & 40 & 5.9395 & .0058 & 19.1151 & .0204 \\
\hline 2 & 30 & 4991 & .0008 & 13.9733 & .0310 & 6 & 50 & 5.8610 & .0058 & 24.4942 & .0241 \\
\hline 2 & 40 & .4998 & .0010 & 18.9934 & .0473 & 6 & 60 & 5.8108 & .0060 & 29.8494 & .0308 \\
\hline 2 & 50 & .5008 & .0013 & 24.0249 & .0759 & 6 & 70 & 5.7688 & .0079 & 35.1205 & .0522 \\
\hline 2 & 60 & .5015 & .0014 & 29.0718 & .0905 & 6 & 80 & 5.7310 & .0075 & 40.3296 & .0565 \\
\hline 2 & 70 & .5011 & .0012 & 34.0281 & .0955 & 6 & 90 & 5.6965 & .0100 & 45.4839 & .0787 \\
\hline 2 & 80 & .5004 & .0011 & 38.9781 & .1139 & 6 & 100 & 5.6622 & .0093 & 50.5601 & .0804 \\
\hline 2 & 90 & .5004 & .0011 & 43.9846 & .1161 & 7 & 30 & 8.0576 & .0122 & 13.4839 & .0210 \\
\hline 2 & 100 & .5004 & .0010 & 48.9952 & .1092 & 7 & 40 & 7.9263 & .0097 & 19.3146 & .0254 \\
\hline 3 & 10 & 1.4615 & .0021 & 3.5677 & .0059 & 7 & 50 & 7.7962 & .0090 & 24.9112 & .0316 \\
\hline 3 & 20 & 1.4391 & .0024 & 8.5942 & .0159 & 7 & 60 & 7.7109 & .0106 & 30.4740 & .0426 \\
\hline 3 & 30 & 1.4264 & .0028 & 13.5165 & .0239 & 7 & 70 & 7.6495 & .0112 & 35.9733 & .0532 \\
\hline 3 & 40 & 1.4165 & .0025 & 18.3722 & .0339 & 7 & 80 & 7.5798 & .0112 & 41.3033 & .0611 \\
\hline 3 & 50 & 1.4126 & .0021 & 23.2346 & .0345 & 7 & 90 & 7.5137 & .0096 & 46.5561 & .0646 \\
\hline 3 & 60 & 1.4117 & .0023 & 28.1393 & .0473 & 7 & 100 & 7.4701 & .0122 & 51.8415 & .0879 \\
\hline 3 & 70 & 1.4081 & .0019 & 32.9634 & .0528 & 8 & 30 & 10.3174 & .0166 & 13.3077 & .0232 \\
\hline 3 & 80 & 1.4041 & .0021 & 37.7299 & .0681 & 8 & 40 & 10.0928 & .0118 & 19.3599 & .0233 \\
\hline 3 & 90 & 1.4004 & .0020 & 42.5085 & .0727 & 8 & 50 & 9.9131 & .0094 & 25.2065 & .0266 \\
\hline 3 & 100 & 1.3970 & .0025 & 47.2469 & .0963 & 8 & 60 & 9.7875 & .0132 & 30.9862 & .0407 \\
\hline 4 & 20 & 2.7390 & .0034 & 8.4389 & .0129 & 8 & 70 & 9.6777 & .0121 & 36.6246 & .0459 \\
\hline 4 & 30 & 2.6979 & .0040 & 13.5666 & .0205 & 8 & 80 & 9.5954 & .0106 & 42.2148 & .0470 \\
\hline 4 & 40 & 2.6678 & .0037 & 18.5891 & .0285 & 8 & 90 & 9.5104 & .0126 & 47.6753 & .0638 \\
\hline 4 & 50 & 2.6509 & .0037 & 23.5816 & .0344 & 8 & 100 & 9.4378 & .0154 & 53.0880 & .0882 \\
\hline 4 & 60 & 2.6440 & .0037 & 28.6169 & .0451 & 9 & 30 & 12.7596 & .0199 & 13.0054 & .0214 \\
\hline 4 & 70 & 2.6273 & .0045 & 33.4731 & .0631 & 9 & 40 & 12.4468 & .0196 & 19.3175 & .0309 \\
\hline 4 & 80 & 2.6101 & .0045 & 38.2554 & .0704 & 9 & 50 & 12.1993 & .0160 & 25.3959 & .0332 \\
\hline 4 & 90 & 2.5971 & .0050 & 43.0491 & .0921 & 9 & 60 & 12.0301 & .0168 & 31.3880 & .0442 \\
\hline 4 & 100 & 2.5879 & .0044 & 47.8435 & .0889 & 9 & 70 & 11.8975 & .0149 & 37.2875 & .0455 \\
\hline 5 & 20 & 4.3336 & .0041 & 8.2193 & .0103 & 9 & 80 & 11.7798 & .0166 & 43.0683 & .0611 \\
\hline 5 & 30 & 4.2409 & .0070 & 13.6075 & .0254 & 9 & 90 & 11.6700 & .0168 & 48.7397 & .0732 \\
\hline 5 & 40 & 4.1901 & .0055 & 18.8849 & .0266 & 9 & 100 & 11.5891 & .0162 & 54.4027 & .0846 \\
\hline 5 & 50 & 4.1460 & .0059 & 24.0506 & .0370 & 10 & 40 & 14.9622 & .0245 & 19.1574 & .0326 \\
\hline 5 & 60 & 4.1227 & .0052 & 29.2296 & .0430 & 10 & 50 & 14.6170 & .0193 & 25.4273 & .0356 \\
\hline 5 & 70 & 4.0901 & .0061 & 34.2507 & .0604 & 10 & 60 & 14.4061 & .0162 & 31.6369 & .0364 \\
\hline 5 & 80 & 4.0630 & .0068 & 39.2278 & .0735 & 10 & 70 & 14.2234 & .0188 & 37.7142 & .0462 \\
\hline 5 & 90 & 4.0428 & .0076 & 44.2211 & .0899 & 10 & 80 & 14.0750 & .0177 & 43.6801 & .0514 \\
\hline 5 & 100 & 4.0242 & .0072 & 49.1511 & .0894 & 10 & 90 & 13.9537 & .0196 & 49.6049 & .0692 \\
\hline 6 & 20 & 6.2146 & .0056 & 7.9360 & .0086 & 10 & 100 & 13.8663 & .0181 & 55.5136 & .0786 \\
\hline
\end{tabular}

"normalized" values yield uniform standard errors for the range in Table 1 . The following estimates for $a$ and $b$ for given $n$ and $k$ were obtained for $r^{2}$ : 


$$
\begin{aligned}
a & \approx 0.5+(k-2)\left[0.656849+0.143161 \sqrt{k-2}-0.0136582 \sqrt{(k-2)^{3}}\right. \\
& \left.+(k-2)\left(0.0981548+\frac{1.8702}{n}-\frac{24.1483}{n^{2}}+\frac{132.632}{n^{3}}\right)\right] \\
b & \approx \frac{n-2}{2}+(k-2)[-13.8256+9.85850 \sqrt{k-2}-1.618145(k-2) \\
& +0.1420267 \sqrt{(k-2)^{3}}+\frac{104.2541-38.0559 \sqrt{k-2}-6.3085(k-2)}{\sqrt{n-2}} \\
& +\frac{-222.772-100.9864 \sqrt{k-2}+91.075(k-2)}{n-2} \\
& \left.+\frac{514.178 \sqrt{k-2}-221.7765(k-2)}{\sqrt{(n-2)^{3}}}\right]
\end{aligned}
$$

\section{Calculating Approximate Fractiles}

Calculating the significance level of a certain value of the multirelation coefficient can be done by the following algorithm:

1. A multirelation coefficient of $r$ was obtained for given values of $k$ and $n$.

2. Estimate the values of $a$ and $b$ using equation (9).

3. Estimate the significance as $1-I_{r^{2}}(a, b)$ where $I_{x}(a, b)$ is the incomplete beta distribution (Abramowitz and Stegun, 1972).

Calculating the critical value of $r$ for a given significance $\alpha$ can be done as a binary search on the segment $[0,1]$ using the algorithm.

We tested this procedure on various values of $n, k$ and $\alpha$ and compared the critical value of $r$ obtained by the algorithm with a simulation of 10,000 matrices. The comparison is reported in Table 2. The simulated fractiles are given in parentheses next to the calculated fractiles.

We know that for $k=2$ the quantity $\frac{r \sqrt{n-2}}{\sqrt{1-r^{2}}}$ is a Student $t$ distribution. We have found that the same quantity is well behaved for the multirelation coefficient fractiles. It can be used to estimate fractiles for values of $n$ which are not reported in Tables 2 and 3. We define these values as the Multirelated $t$ Fractiles. In Table 3 we give the calculated values for these Multirelated $t$ fractiles.

\section{An Example}

In Drezner (1995) an example taken from Kendall (1980) was used to demonstrate the concept of the multirelation coefficient. Fifteen traits of applicants were tested for relationships. The traits were: 
Table 2. Fractiles of the multirelation coefficient and simulation results

\begin{tabular}{|c|c|c|c|c|c|c|}
\hline$k$ & $n$ & 0.90 & 0.95 & 0.975 & 0.99 & 0.995 \\
\hline 2 & 10 & $.549(.552)$ & $632(.630)$ & $.697(.697)$ & $.765(.769)$ & $.805(.815)$ \\
\hline 2 & 20 & $.378(.380)$ & $.444(.441)$ & $.499(.494)$ & $.561(.552)$ & $.602(.596)$ \\
\hline 2 & 30 & $.306(.308)$ & $.361(.361)$ & $.409(.402)$ & $.463(.450)$ & $.499(.489)$ \\
\hline 2 & 40 & $.264(.262)$ & $.312(.311)$ & $.354(.353)$ & $.403(.403)$ & $.435(.431)$ \\
\hline 2 & 50 & $.235(.233)$ & $.279(.276)$ & $.317(.317)$ & $.361(.359)$ & $.391(.384)$ \\
\hline 2 & 100 & $.166(.166)$ & $.197(.199)$ & $.224(.227)$ & $.257(.263)$ & $.279(.285)$ \\
\hline 3 & 10 & $.746(.744)$ & $.800(.797)$ & $.840(.843)$ & $.880(.886)$ & $.903(.907)$ \\
\hline 3 & 20 & $.540(.544)$ & $.592(.600)$ & $.636(.644)$ & $.684(.690)$ & $.715(.722)$ \\
\hline 3 & 30 & $.443(.443)$ & $.490(.490)$ & $.530(.534)$ & $.575(.582)$ & $.605(.611)$ \\
\hline 3 & 40 & $.386(.385)$ & $.428(.428)$ & $.465(.465)$ & $.506(.510)$ & $.534(.539)$ \\
\hline 3 & 50 & $.346(.343)$ & $.385(.385)$ & $.418(.419)$ & $.457(.455)$ & $.483(.481)$ \\
\hline 3 & 100 & $.246(.246)$ & $.274(.275)$ & $.300(.302)$ & $.329(.329)$ & $.349(.349)$ \\
\hline 4 & 20 & $.644(.644)$ & $.687(.688)$ & $.722(.726)$ & $.760(.768)$ & $.785(.789)$ \\
\hline 4 & 30 & $.537(.536)$ & $.577(.578)$ & $.612(.615)$ & $.650(.652)$ & $.675(.683)$ \\
\hline 4 & 40 & $.471(.472)$ & $.508(.510)$ & $.540(.542)$ & $.577(.583)$ & $.601(.614)$ \\
\hline 4 & 50 & $.424(.423)$ & $.459(.458)$ & $.489(.490)$ & $.524(.527)$ & $.547(.547)$ \\
\hline 4 & 100 & $.305(.305)$ & $.332(.331)$ & $.355(.355)$ & $.382(.388)$ & $.401(.405)$ \\
\hline 5 & 20 & $.721(.723)$ & $.756(.757)$ & $.785(.787)$ & $.815(.814)$ & $.835(.834)$ \\
\hline 5 & 30 & $.609(.607)$ & $.644(.641)$ & $.673(.673)$ & $.706(.705)$ & $.728(.728)$ \\
\hline 5 & 40 & $.536(.537)$ & $.570(.571)$ & $.598(.600)$ & $.631(.634)$ & $.652(.654)$ \\
\hline 5 & 50 & $.485(.487)$ & $.517(.521)$ & $.544(.554)$ & $.575(.584)$ & $.596(.607)$ \\
\hline 5 & 100 & $.353(.352)$ & $.378(.377)$ & $.400(.401)$ & $.425(.426)$ & $.442(.441)$ \\
\hline 6 & 20 & $.780(.780)$ & $.809(.813)$ & $.832(.838)$ & $.857(.863)$ & $.873(.878)$ \\
\hline 6 & 30 & $.666(.666)$ & $.697(.700)$ & $.723(.727)$ & $.751(.754)$ & $.770(.775)$ \\
\hline 6 & 40 & $.590(.591)$ & $.621(.621)$ & $.646(.649)$ & $.675(.679)$ & $.694(.700)$ \\
\hline 6 & 50 & $.536(.539)$ & $.565(.568)$ & $.590(.593)$ & $.618(.626)$ & $.637(.646)$ \\
\hline 6 & 100 & $.393(.392)$ & $.417(.419)$ & $.437(.442)$ & $.461(.468)$ & $.477(.486)$ \\
\hline 7 & 30 & $.714(.715)$ & $.741(.742)$ & $.764(.765)$ & $.789(.793)$ & $.805(.811)$ \\
\hline 7 & 40 & $.636(.639)$ & $.663(.666)$ & $.686(.692)$ & $.712(.723)$ & $.729(.740)$ \\
\hline 7 & 50 & $.579(.581)$ & $.606(.607)$ & $.629(.630)$ & $.654(.657)$ & $.671(.673)$ \\
\hline 7 & 100 & $.428(.428)$ & $.450(.453)$ & $.470(.473)$ & $.492(.497)$ & $.507(.514)$ \\
\hline 8 & 30 & $.754(.753)$ & $.778(.780)$ & $.798(.802)$ & $.820(.823)$ & $.834(.837)$ \\
\hline 8 & 40 & $.676(.676)$ & $.700(.703)$ & $.721(.725)$ & $.744(.749)$ & $.760(.764)$ \\
\hline 8 & 50 & $.617(.618)$ & $.642(.644)$ & $.663(.664)$ & $.686(.688)$ & $.702(.706)$ \\
\hline 8 & 100 & $.459(.458)$ & $.480(.482)$ & $.499(.500)$ & $.520(.524)$ & $.534(.538)$ \\
\hline 9 & 30 & $.787(.789)$ & $.809(.810)$ & $.826(.829)$ & $.845(.849)$ & $.858(.863)$ \\
\hline 9 & 40 & $.710(.710)$ & $.733(.734)$ & $.751(.754)$ & $.773(.779)$ & $.786(.793)$ \\
\hline 9 & 50 & $.651(.651)$ & $.674(.677)$ & $.693(.698)$ & $.714(.721)$ & $.729(.739)$ \\
\hline 9 & 100 & $.487(.487)$ & $.507(.507)$ & $.525(.527)$ & $.545(.550)$ & $.559(.567)$ \\
\hline 10 & 40 & $.740(.742)$ & $.761(.764)$ & $.778(.781)$ & $.797(.802)$ & $.809(.814)$ \\
\hline 10 & 50 & $.681(.682)$ & $.702(.703)$ & $.720(.722)$ & $.740(.741)$ & $.753(.752)$ \\
\hline 10 & 100 & $.513(.512)$ & $.532(.532)$ & $.549(.552)$ & $.568(.570)$ & $.581(.586)$ \\
\hline
\end{tabular}

(1) Form of Letter of application

(2) Appearance

(3) Academic Ability

(4) Likability

(5) Self-confidence
(6) Lucidity

(7) Honesty

(8) Salesmanship

(9) Experience

(10) Drive
(11) Ambition

(12) Grasp

(13) Potential

(14) Keenness to join

(15) Suitability 
Table 3. Fractiles of the Multirelated $t$ distribution

\begin{tabular}{|r|r|r|r|r|r|r|}
\hline$k$ & $n$ & 0.90 & 0.95 & 0.975 & 0.99 & 0.995 \\
\hline 2 & 10 & 1.860 & 2.306 & 2.752 & 3.356 & 3.833 \\
2 & 20 & 1.735 & 2.101 & 2.445 & 2.879 & 3.197 \\
2 & 30 & 1.702 & 2.049 & 2.369 & 2.764 & 3.047 \\
2 & 40 & 1.687 & 2.025 & 2.334 & 2.712 & 2.981 \\
2 & 50 & 1.678 & 2.011 & 2.315 & 2.683 & 2.943 \\
2 & 100 & 1.662 & 1.985 & 2.277 & 2.628 & 2.873 \\
3 & 10 & 3.169 & 3.766 & 4.380 & 5.240 & 5.939 \\
3 & 20 & 2.719 & 3.119 & 3.497 & 3.980 & 4.338 \\
3 & 30 & 2.617 & 2.976 & 3.308 & 3.721 & 4.020 \\
3 & 40 & 2.577 & 2.920 & 3.233 & 3.618 & 3.894 \\
3 & 50 & 2.555 & 2.889 & 3.192 & 3.562 & 3.825 \\
3 & 100 & 2.508 & 2.824 & 3.108 & 3.450 & 3.689 \\
4 & 20 & 3.572 & 4.011 & 4.429 & 4.966 & 5.368 \\
4 & 30 & 3.369 & 3.743 & 4.090 & 4.523 & 4.838 \\
4 & 40 & 3.288 & 3.637 & 3.957 & 4.351 & 4.633 \\
4 & 50 & 3.246 & 3.582 & 3.887 & 4.260 & 4.526 \\
4 & 100 & 3.168 & 3.480 & 3.760 & 4.096 & 4.333 \\
5 & 20 & 4.409 & 4.899 & 5.367 & 5.973 & 6.429 \\
5 & 30 & 4.060 & 4.453 & 4.820 & 5.278 & 5.612 \\
5 & 40 & 3.919 & 4.276 & 4.605 & 5.010 & 5.300 \\
5 & 50 & 3.847 & 4.187 & 4.496 & 4.874 & 5.143 \\
5 & 100 & 3.730 & 4.039 & 4.317 & 4.650 & 4.884 \\
6 & 20 & 5.283 & 5.837 & 6.371 & 7.065 & 7.589 \\
6 & 30 & 4.730 & 5.148 & 5.538 & 6.026 & 6.383 \\
6 & 40 & 4.510 & 4.879 & 5.218 & 5.637 & 5.938 \\
6 & 50 & 4.400 & 4.745 & 5.060 & 5.445 & 5.719 \\
6 & 100 & 4.230 & 4.538 & 4.813 & 5.144 & 5.376 \\
7 & 30 & 5.400 & 5.847 & 6.264 & 6.789 & 7.173 \\
7 & 40 & 5.083 & 5.467 & 5.819 & 6.255 & 6.568 \\
7 & 50 & 4.926 & 5.279 & 5.600 & 5.994 & 6.275 \\
7 & 100 & 4.688 & 4.995 & 5.270 & 5.599 & 5.831 \\
8 & 30 & 6.077 & 6.556 & 7.006 & 7.572 & 7.987 \\
8 & 40 & 5.650 & 6.050 & 6.418 & 6.874 & 7.202 \\
8 & 50 & 5.437 & 5.800 & 6.130 & 6.535 & 6.824 \\
8 & 100 & 5.117 & 5.423 & 5.697 & 6.027 & 6.258 \\
9 & 30 & 6.759 & 7.275 & 7.759 & 8.371 & 8.821 \\
9 & 40 & 6.216 & 6.635 & 7.021 & 7.499 & 7.845 \\
9 & 50 & 5.942 & 6.316 & 6.657 & 7.075 & 7.373 \\
9 & 100 & 5.523 & 5.830 & 6.105 & 6.435 & 6.666 \\
10 & 40 & 6.782 & 7.222 & 7.628 & 8.131 & 8.495 \\
10 & 50 & 6.444 & 6.831 & 7.184 & 7.617 & 7.926 \\
10 & 100 & 5.913 & 6.222 & 6.498 & 6.829 & 7.061 \\
\hline
\end{tabular}

The data consisted of 48 applicants and the correlation matrix between these traits is given (Drezner, 1995; Kendall, 1980). Note that two entries should be 
corrected in Drezner (1995): $r_{5,14}=0.48, r_{11,15}=0.43$. A procedure similar to backward step-wise regression was presented in Drezner (1995). Variables which are not associated with the rest were dropped one by one according to a certain rule. The issue of which of the subsets is best remained unresolved. In order to determine which of the subsets is the most significant, we need to calculate the significance level of the multirelation coefficient for each subset. The present paper provides us with the tools necessary to make such determination. In Table 4 we give the original subsets presented in Drezner (1995) with their calculated multirelation coefficients as well as the significance level of each multirelation coefficient calculated by the method presented in this paper. Other methods for subset selection may be considered as well, possibly yielding better results.

By examining Table 4 it is clear that the best subset is the subset of 6 variables (Likability, Self-confidence, Lucidity, Honesty, Ambition, Grasp) because it yields the best significance level.

Since this particular problem with $k=15$ has only $2^{15}-16=32,752$ possible subsets (excluding subsets of less than two members), it is feasible to calculate the multirelation coefficient for each subset and select the best one. In Table 5 we report the best multirelation coefficient for subsets of $2,3, \ldots, 15$ elements, a list of the members of that subset, and the corresponding significance. Note that the significance levels are quite small. However, these values should be quite accurate because both the beta distribution and the theoretical multirelation distribution are anchored to zero at both ends of the segment $[0,1]$. Such small values of significance cannot be verified by simulation. Some of the groups have an improved significance level. However, the best group obtained by this analysis is still the same group of 6 variables. We conclude that the step-wise backward procedure is effective.

\section{Appendix}

In Johnson and Kotz (1972) there are some simplified formulas when all the correlation coefficients are equal to each other (and in our case they are all equal to $\left.-\frac{1}{k-1}\right)$. The case when all the $\rho$ 's are positive is relatively simple. Define $\Phi(h, k, \rho)=\operatorname{Pr}\left(x_{i} \geq h\right.$, for $\left.i=1, \ldots, k\right)$ when the correlation coefficient between $X_{i}$ and $X_{j}$ is equal to $\rho$ for all $i \neq j . \quad k=1$ represents the univariate Normal distribution: $\Phi(h)=\Phi(h, 1, \rho)$ for any $\rho$. For $\rho \geq 0$ (Johnson and Kotz 1972):

$$
\Phi(h, k, \rho)=\int_{-\infty}^{\infty} Z(u)\left\{\Phi\left[\frac{h-u \sqrt{\rho}}{\sqrt{1-\rho}}\right]\right\}^{k} d u
$$

where $Z(X)$ is the standard normal density function. The integral (11) can be calculated using Gaussian quadrature formulas based on Hermite polynomials (Abramowitz and Stegun, 1972).

For a negative $\rho$ a recursion formula by $k$ is given: 
Table 4. Significance levels for the example problem

\begin{tabular}{|c|c|c|}
\hline$k$ & $r$ & Significance \\
\hline 2 & 0.88000 & $1.77 \cdot 10^{-16}$ \\
3 & 0.89699 & $6.18 \cdot 10^{-16}$ \\
4 & 0.92007 & $4.26 \cdot 10^{-17}$ \\
5 & 0.93376 & $6.46 \cdot 10^{-18}$ \\
6 & 0.95047 & $7.91 \cdot 10^{-20}$ \\
7 & 0.95232 & $2.67 \cdot 10^{-19}$ \\
8 & 0.95475 & $7.36 \cdot 10^{-19}$ \\
9 & 0.96102 & $2.49 \cdot 10^{-19}$ \\
10 & 0.96332 & $6.80 \cdot 10^{-19}$ \\
11 & 0.96394 & $5.11 \cdot 10^{-18}$ \\
12 & 0.96539 & $2.11 \cdot 10^{-17}$ \\
13 & 0.96544 & $1.83 \cdot 10^{-16}$ \\
14 & 0.96553 & $1.24 \cdot 10^{-15}$ \\
15 & 0.96559 & $6.46 \cdot 10^{-15}$ \\
\hline
\end{tabular}

Table 5. Best significance levels for the example problem

\begin{tabular}{|c|c|l|l|l|}
\hline$k$ & $r$ & \multicolumn{1}{|c|}{ Set } & Significance \\
\hline 2 & 0.88000 & 612 & & $1.77 \cdot 10^{-16}$ \\
3 & 0.91419 & 61213 & $1.31 \cdot 10^{-17}$ \\
4 & 0.92367 & 6101213 & $1.59 \cdot 10^{-17}$ \\
5 & 0.93789 & 410121314 & $1.58 \cdot 10^{-18}$ \\
6 & 0.95047 & 45671112 & $7.91 \cdot 10^{-20}$ \\
7 & 0.95305 & 145671112 & $1.89 \cdot 10^{-19}$ \\
8 & 0.95725 & 456710121314 & $2.02 \cdot 10^{-19}$ \\
9 & 0.96102 & 45671011121314 & $2.49 \cdot 10^{-19}$ \\
10 & 0.96332 & 4567101112131415 & $6.80 \cdot 10^{-19}$ \\
11 & 0.96445 & 34567101112131415 & $3.71 \cdot 10^{-18}$ \\
12 & 0.96539 & 345678101112131415 & $2.11 \cdot 10^{-17}$ \\
13 & 0.96548 & 3456789101112131415 & $1.78 \cdot 10^{-16}$ \\
14 & 0.96553 & 13456789101112131415 & $1.24 \cdot 10^{-15}$ \\
15 & 0.96559 & 123456789101112131415 & $6.46 \cdot 10^{-15}$ \\
\hline
\end{tabular}




$$
\Phi(h, k, \rho)=\sum_{i=1}^{k}(-1)^{i+1}\left(\begin{array}{c}
k \\
i
\end{array}\right) \Phi\left(\alpha h, i, \rho^{\prime}\right) \Phi(h, k-i, \rho)
$$

where $\alpha=\sqrt{\frac{1-\rho}{[1+(k-1) \rho][1+(k-2) \rho]}}, \rho^{\prime}=\frac{-\rho}{1+(k-2) \rho}$.

When $\rho=-\frac{1}{k-1}$ then $\alpha$ is undefined and therefore we need to find it as a limit. $\rho^{\prime}$ approaches 1 and $\alpha$ approaches infinity, therefore, for a negative $h \Phi\left(\alpha h, k, \rho^{\prime}\right)$ approaches 1. In conclusion, when $\rho=-\frac{1}{k-1}, \Phi\left(\alpha h, k, \rho^{\prime}\right)=1$. We get:

$$
\Phi\left(h, k,-\frac{1}{k-1}\right)=\sum_{i=1}^{k}(-1)^{i+1}\left(\begin{array}{c}
k \\
i
\end{array}\right) \Phi\left(h, k-i,-\frac{1}{k-1}\right)
$$

This result is true only for the last stage of the recursion formula. For lower values of $k$ the recursion formula (12) can be calculated without undefined values.

For a positive $h, \Phi\left(\alpha h, k, \rho^{\prime}\right)=0$, and equation (13) is no longer true. The probability $\Phi\left(h, k,-\frac{1}{k-1}\right)=0$. This is in line with the observation that the smallest $\lambda$ cannot be greater than 1 because a positive $h$ means that all eigenvalues are greater than 1 which is impossible.

Calculating the required multinormal probabilities using this approach is very efficient even for large values of $k$.

\section{Notes}

1. We are thankful to D. Aigner of University of California-Irvine for turning our attention to this discussion

\section{References}

1. Abramowitz, M. and I.A. Stegun. Handbook of Mathematical Functions, Dover Publications Inc., New York, 1972.

2. Drezner, Z. Computation of the Multivariate Normal Integral. ACM Transactions on Mathematical Software, 18, 470-480, 1992.

3. Drezner, Z. Multirelation - A Correlation Among More Than Two Variables. Computational Statistics and Data Analysis, 19, 283-292, 1995.

4. Johnson, N.L. and S. Kotz. Distributions In Statistics: Continuous Multivariate Distributions, John Wiley \& Sons, Inc., New York, 1972.

5. Kendall, M.G. and A. Stuart. The Advanced Theory of Statistics, V. Distribution Theory, 2nd Edition, p.396, Hafner Publishing Co., New York, 1963.

6. Kendall, M.G. and A.S. Stuart. The advanced Theory of Statistics, Vol. 3 Hafner Publishing Co., New York, 1966.

7. Kendall, M.G. Multivariate Analysis 2nd Ed., MacMillan Publishing, New York, 1980.

8. Law A.M. and W.D. Kelton. Simulation Modeling \& 8 Analysis, 2nd Ed., McGraw Hill, New York, 1991.

9. Marse K. and S.D. Roberts. Implementing a Portable FORTRAN Uniform $(0,1)$ Generator. Simulation, 41, 135-139, 1983.

10. Stuart A. and J.K. Ord. Kendall's Advanced Theory of Statistics Vol. 2, Fifth Edition, Edward Arnold, A division of Hodder \& Stoughton, London, 1991. 


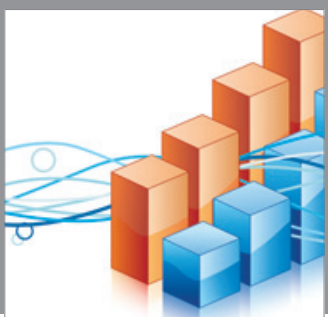

Advances in

Operations Research

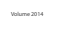

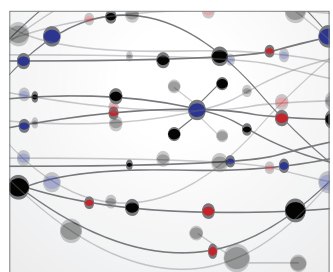

\section{The Scientific} World Journal
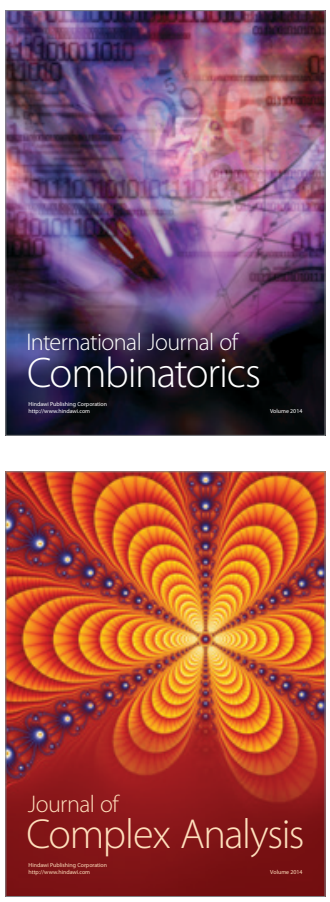

International Journal of

Mathematics and

Mathematical

Sciences
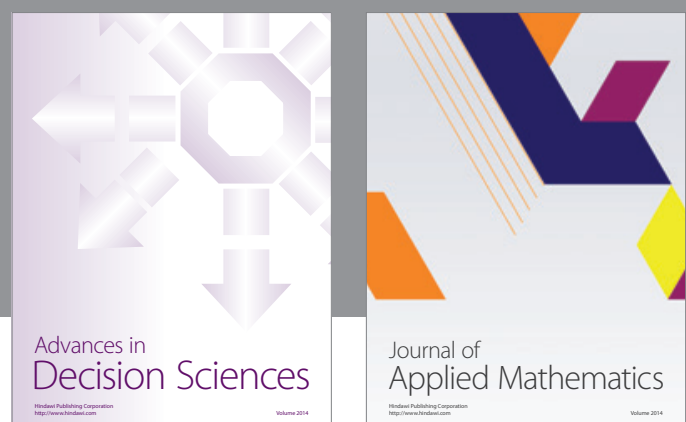

Journal of

Applied Mathematics
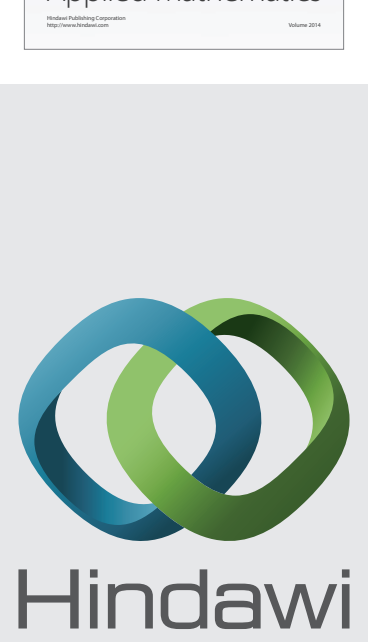

Submit your manuscripts at http://www.hindawi.com
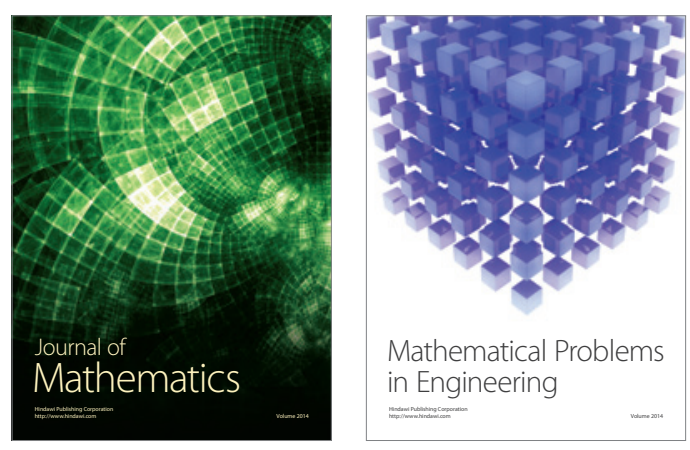

Mathematical Problems in Engineering


Journal of

Function Spaces
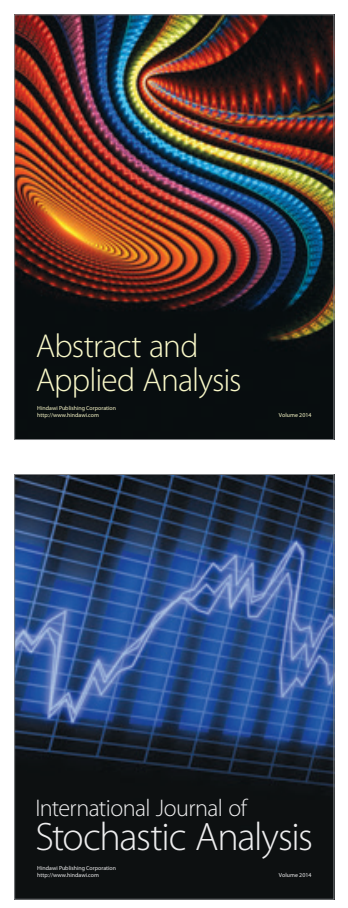

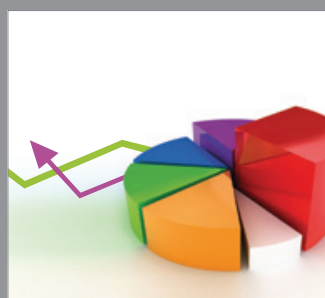

ournal of

Probability and Statistics

Promensencen
